\title{
Desempeño fonético-fonológico en niños con Trastorno del Espectro Autista (TEA) de pre-kínder a tercero básico
}

\author{
Phonetic and phonological performance in \\ children with Autism Spectrum Disorder (ASD) \\ from pre-primary school to third grade
}

\section{Juan Carlos Torres \\ Departamento de Español Universidad de Concepción}

\section{Hernán León}

Departamento de Fonoaudiología Universidad de Concepción

Mauricio Figueroa

Departamento de Español Universidad de Concepción

\footnotetext{
Contacto con el autor: Hernán León V. Concepción - Chile Correo-e: hleon@udec.cl
}

Recibido: $13 / 04 / 2018$ Aceptado: 09/07/2018

\section{RESUMEN}

Desde el punto de vista lingüístico, son conocidas las repercusiones del Trastorno del Espectro Autista (TEA) en la comunicación y el lenguaje, sin embargo, existen pocos estudios específicos para el nivel Fonético-Fonológico. El objetivo de este estudio fue analizar el desempeño Fonético-Fonológico de niños con TEA de los niveles pre-kínder, kínder, primero, segundo y tercero básico, mediante el uso de la Clasificación de Ajustes Fonético-Fonológicos del habla infantil (CLAFF). Para ello, se utilizó un enfoque descriptivo transversal y un diseño no probabilístico de tipo consecutivo. Los principales resultados mostraron que los niños con TEA realizan como Ajuste Fonético-Fonológico (AFF) a los rasgos del fonema el Ajuste de Modo y en los AFF a la sílaba, la Síncopa. Además, mediante la prueba de chi-cuadrado se determinó que existía una asociación entre las variables Nivel escolar y Categoría de ajuste. Para investigar más a fondo esta relación, se procedió a realizar un análisis de Regresión Logística Multinomial (RLM), el que reveló que Nivel escolar predice la probabilidad de aparición de AFF que afecten al Rasgo, en oposición a Sílaba, en los niveles estudiados.

Palabras clave: fonética, fonología, autismo, TEA, CLAFF.

\section{ABSTRACT}

From a linguistic standpoint, the consequences of Autism Spectrum Disorder (ASD) in both communication and linguistic skills are relatively well known. However, few studies have been conducted to observe the phonetic and phonemic domain. The aim of this study is to analyse the phonetic and phonemic performance of children with ASD from pre-primary school to third grade. This was accomplished by using the CLAFF, a guideline for classifying phonetic and phonemic adjustments. This study presents a cross-sectional approach and the sample was obtained via a consecutive non-probabilistic model. The main results showed that children with ASD prefer adjustments in manner of articulation and syncope. A Chi-squared analysis revealed a significant statistical association between School grade and Category of adjustment. To further observe this association, a multinomial logistic regression analysis was run, which showed that School grade predicted the probability of occurrence of adjustments that affect phonemic features rather the syllable.

Keywords: phonetics, phonology, autism, ASD, CLAFF. 


\section{Introducción}

Desde las primeras definiciones de Kanner (1943)

y Asperger (1944), la conceptualización sobre autismo ha variado considerablemente hasta llegar a lo que hoy se establece en el manual diagnóstico DSM-V. Este, clasifica al autismo entre los trastornos del neurodesarrollo y lo denomina Trastorno del Espectro Autista (TEA), distinguiendo como condiciones esenciales para su diagnóstico la existencia de un déficit social y de comunicación junto a la presencia de intereses fijos $y$ comportamientos repetitivos (American Psychiatric Association, 2014).

Si bien las manifestaciones del TEA son variadas (Fortea, Escandell, \& Castro, 2014; L. Wing \& Gould, 1979; Lorna Wing, 1997), la mayoría de los autores concuerdan en que el TEA se presenta con afectación del lenguaje (American Psychiatric Association, 2002; Gerber, 2003; Irarrázaval, Brokering, \& Murillo, 2005; McCleery, Tully, Slevc, \& Schreibman, 2006; Organización Mundial de la Salud, 1992; Rodríguez, 1988; Seung, 2007; Wing \& Gould, 1979; Wing, 1997). Sin embargo, esta afectación varía en el grado con el que se presenta, ya que puede manifestar desde una afectación severa con completa ausencia del lenguaje, hasta una aparente ausencia de déficit (Whitehouse, Barry, \& Bishop, 2008). Además, considerando el lenguaje por niveles, se ha confirmado que el nivel del lenguaje más afectado en las personas con TEA es el nivel pragmático (Dioses et al., 2014).

La mayoría de los estudios acerca del desempeño fonético-fonológico en niños con TEA han sido realizados en la lengua inglesa. Estos estudios no han llegado a consenso sobre el grado de alteración de este nivel en niños con TEA. Al respecto, Wing (1998) menciona que las dificultades de habla son comunes en personas con TEA y pueden variar en grado de afectación, en cambio Happé (1998), señala que la fonología en niños con TEA sería bastante normal. En la misma línea, McCann, Peppé, Gibbon, O’Hare, \& Rutherford (2007), señalan que en general los sujetos con autismo no presentan dificultades en la articulación de sonidos lingüísticos. Por su parte, McCleery et al. (2006), en un estudio comparativo de niños con TEA y afectación severa del lenguaje y niños con desarrollo típico del lenguaje, informan que si bien en los niños con autismo el desarrollo fonológico sigue un patrón de desarrollo normal, este se encuentra retrasado. En un estudio realizado por Rapin, Dunn, Allen, Stevens, \& Fein (2009) se describió la existencia de dos tipos de desórdenes lingüísticos en niños con TEA en edad escolar. Un primer tipo, compuesto por un $24 \%$ de la muestra total, presentaba habilidades fonológicas expresivas severamente afectadas a la edad de 8 años y 6 meses, y un segundo tipo, compuesto por el $76 \%$ de la muestra, presentaba una fonología expresiva que iba desde rendimiento límite/bajo promedio a rendimiento superior al promedio.

Otro estudio en niños con Autismo de alto funcionamiento y Síndrome de Asperger de habla inglesa, reportó que sólo un $12 \%$ de la muestra presentaba puntajes bajo la norma (Cleland, Gibbon, Peppé, O'Hare, \& Rutherford, 2010), evidenciando un desorden o retraso del habla, mientras que el resto de los informantes presentaba puntajes que los ubicaban en rango de normalidad. Sin embargo, en el grupo con rendimiento normal, un $33 \%$ presentaba 
errores en su producción (Cleland et al., 2010). Contabilizando a los niños dentro y fuera de la norma, un $41 \%$ de la muestra evidenció distorsiones fonético-fonológicas en su discurso, y de estas, un porcentaje no menor fue caracterizado como procesos fonológicos del desarrollo, aunque también fueron encontradas distorsiones que no son propias del desarrollo fonético-fonológico. Estas distorsiones fueron encontradas tanto en niños con puntaje bajo el rango normal como en niños con puntaje sobre la norma. Lo anterior coincide con otros estudios previamente realizados en adolescentes y adultos con dificultades similares, que han concluido que estas distorsiones no se resuelven con el tiempo (Cleland et al., 2010). Adicionalmente, los autores señalan que, si bien los desórdenes del habla pueden estar relacionados o no específicamente con el autismo, éstos añaden barreras comunicativas y sociales, por lo que deben ser diagnosticados $y$ tratados a tiempo.

El estudio del nivel fonético-fonológico en personas con TEA no ha sido la primera opción al momento de iniciar nuevas investigaciones (McCann et al., 2007). Ello debido, por un lado, a que existe acuerdo en que las principales afectaciones del lenguaje en personas con TEA son de tipo pragmático y no fonético-fonológico (Dioses et al., 2014) y por otro, a la gran variedad de afectación de otras habilidades lingüísticas. Sin embargo, como ya se indicó, las dificultades del habla en esta población pueden constituir un impedimento importante en el logro de un mejor desempeño social y escolar, por ello se hace necesario realizar más estudios que contribuyan al conocimiento del desempeño de este grupo en dicho nivel (McCleery et al., 2006).
El presente trabajo se enmarca en una línea de investigación que utiliza la pauta CLAFF (Soto-Barba, León, \& Torres, 2011), para describir los ajustes fonético-fonológicos realizados por niños chilenos. Entenderemos como un Ajuste Fonético-Fonológico (AFF) "cualquier realización del habla infantil que no coincide con el modelo fonológico del español estándar, como ocurre en ['lo.xo] por ['ro.xo]". Es posible distinguir dos categorías de ajustes, la de los que afectan a los rasgos de los segmentos sonoros y la de los que afectan a la sílaba, cada una de estas categorías se subdivide en diferentes tipos de ajuste, según la modificación que se produzca al rasgo o a la estructura de la sílaba (Soto-Barba et al., 2011). La pauta CLAFF ha demostrado ser una herramienta útil para describir y cuantificar el desempeño fonéticofonológico infantil, lo que ha permitido el estudio de estos niveles en diferentes poblaciones infantiles: (1) niños con desarrollo típico (León, 2012), (2) niños que aprenden inglés como segunda lengua (Fuica \& Soto-Barba, 2014), (3) niños con diagnóstico de TEL Mixto (Torres, Soto-Barba, Soto-Barba, \& Universidad de Concepción, 2016) y (4) niños preescolares en situación de vulnerabilidad (Hamdan, 2017). Considerando que la información disponible en relación con las dificultades fonéticofonológicas en niños con TEA proviene fundamentalmente de investigaciones realizadas en el habla inglesa, adquiere especial relevancia aportar al conocimiento de dicho fenómeno en niños hablantes de español con diagnóstico de TEA.

Esta investigación tiene como objetivo principal describir el desempeño fonético-fonológico de niños con Trastorno del Espectro Autista de los niveles prekínder, kínder, primero, segundo y tercero básicos 
que se encuentran en Programas de Integración Escolar (PIE) de establecimientos educacionales dependientes del Departamento de Administración de Educación Municipal (DAEM) de la comuna de Talcahuano, mediante el uso de la Clasificación de Ajustes Fonético-Fonológicos del habla infantil (CLAFF).

\section{Metodología}

Este estudio se llevó a cabo con un alcance descriptivo, enfoque cuantitativo y diseño transversal. La población estudiada corresponde a 25 niños y niñas entre 4 años 4 meses y 10 años 3 meses de edad, con diagnóstico neurológico de Trastorno del Espectro Autista. Un total de 23 de los 25 participantes eran de género masculino, lo que correspondió a un $92 \%$ de la muestra total, mientras que solo 2 de los participantes, ( $8 \%$ restante) correspondía al género femenino. Se consideraron estudiantes de los niveles escolares pre-kínder, kínder, primero, segundo y tercero básico de establecimientos educacionales dependientes del DAEM de la comuna de Talcahuano y que además, participaban en los Programas de Integración Escolar (PIE) de sus establecimientos. Un $12 \%$ de los participantes pertenecía al nivel pre-kínder (3 participantes), un $12 \%$ al nivel kínder (3 participantes), un $32 \%$ al nivel primero básico ( 8 participantes), un $20 \%$ al nivel segundo básico (5 participantes) y finalmente, un $24 \%$ al nivel de tercero básico (6 participantes).

La selección de los participantes se llevó a cabo a través de un muestreo no probabilístico de tipo consecutivo, incluyendo a todos los sujetos disponibles que presentaran los criterios de inclusión para la muestra. Los criterios de inclusión de los participantes fueron haber sido diagnosticados con TEA por un neurólogo, cursar en alguno de los niveles escolares mencionados y corresponder a los casos clasificados como "verbales" dentro del espectro autista, es decir, que presenten conductas lingüísticas posibles de analizar (para un uso similar del término y definiciones, véase Westerveld \& Roberts (2017). Fueron considerados como criterios de exclusión la existencia de alteraciones anatómicas severas de los órganos fonoarticulatorios o alteraciones sensoriales.

Los participantes pertenecían a establecimientos que presentan en promedio un Índice de Vulnerabilidad (IVE-SINAE) de 76,7\%, por lo que se trata de una población infantil de alta vulnerabilidad, condición que al manifestarse por igual en todos los participantes permitió homologar la procedencia sociocultural. Cabe señalar que, en el presente estudio, no se evaluó el efecto de la variable género en los AFF, pues en este nivel de escolaridad no se aprecian diferencias significativas en el desempeño fonético-fonológico (Pavez, Maggiolo, Peñaloza, \& Coloma, 2009).

La toma de muestras se realizó a través de la aplicación de la prueba TREFF (Test de Repetición Fonético-Fonológica), que consiste en una prueba de repetición directa de 90 ítems, diseñada para el estudio de Hamdan (2017) como un instrumento para elicitar la producción de los alófonos de los fonemas del español de Chile en todos los contextos fonéticos y sus posibilidades combinatorias. En forma previa a la investigación, se pilotearon distintas metodologías en dos integrantes de la 
muestra, determinándose que la prueba TREFF era la que contribuía a elicitar mayor cantidad de elementos a analizar, de manera rápida y sencilla.

Antes de evaluar a los participantes, se solicitó la firma de un consentimiento informado por sus padres o apoderados (Anexo 1). Cabe señalar que, para todos sus efectos, esta investigación cuenta con la aprobación del Comité de Ética de la Dirección de Investigación de la Universidad de Concepción (Anexo 2).

Una vez conformada la muestra, la captura del corpus se realizó en las salas acondicionadas para la atención de los estudiantes pertenecientes a los Programas de Integración Escolar de los respectivos establecimientos educacionales. En una primera instancia, se aplicó la pauta de evaluación de órganos fonoarticulatorios, y luego, a quienes aprobaban dicha evaluación, la prueba TREFF. Durante la realización de las evaluaciones se minimizaron los estímulos distractores visuales y auditivos. La aplicación de TREFF fue registrada con una grabadora digital Tascam DR-40, configurada para una frecuencia de muestreo de $44.1 \mathrm{kHz}$, en formato WAV mono, a una profundidad de 16 bit. Posteriormente, las palabras elicitadas se extrajeron de las señales con el software Praat (Boersma \& Weenink, 2018). Para ello, se ejecutó un script que tenía el objetivo de demarcar los períodos silentes de la grabación, con el fin de aislar y etiquetar cada palabra producida. Como resultado, se etiquetaron los 90 ítems evaluados de cada uno de los 25 informantes, mediante transcripción ortográfica. Una vez finalizado el etiquetado, se aplicó otro script que cumplía la función de recortar cada una de las palabras etiquetadas obteniéndose 90 archivos de audio independientes que portaban el nombre del estímulo producido por el informante. Luego, se seleccionaron las palabras portadoras de AFF, las que fueron analizadas mediante la aplicación de la pauta CLAFF. Este análisis considera un análisis fonético auditivo y la transcripción fonética correspondiente, para finalmente proceder a la clasificación de cada ajuste detectado según la tipología de CLAFF. Una vez efectuado este análisis, la información se vació a una hoja de cálculo con el fin de graficar los datos y establecer medidas de tendencia central para la muestra. Para realizar el análisis, se consideró, primero, la variable Categoría de ajuste, que divide los Ajustes Fonético-Fonológicos (AFF) en dos categorías, el de AFF al Rasgo y AFF a la Sílaba, siguiendo el modelo de análisis utilizado por (León, 2012). En segundo lugar, se consideró la variable Nivel Escolar (pre-kínder a tercero básico) lo que se plasmó en una tabla de contingencias (ver Tabla 1). El análisis estadístico se realizó utilizando el programa R (R Core Team, 2018), en el cual se aplicó inicialmente la prueba chi-cuadrado para explorar la asociación entre estas dos variables. Posteriormente, con el fin de realizar un análisis de mayor profundidad, se realizó un análisis de Regresión Logística Multinomial (RLM) (Tabla 2).

\section{Resultados}

En una primera revisión de los datos, considerando los 25 niños estudiados, se observa un total de 460 Ajustes Fonético-Fonológicos (AFF). Individualmente cada participante produjo un promedio de 18,4 AFF. En relación con las categorías de AFF (Figura 1), 233 AFF afectaron a los rasgos, 
representando un 51\% del total, y 227 AFF afectaron a la estructura de la Sílaba, lo que representa un $49 \%$ del total de los ajustes producidos.

\section{SEGMENTOS O SÍLABAS}

- AFF a los Rasgos $\quad$ AFF a las Sílabas

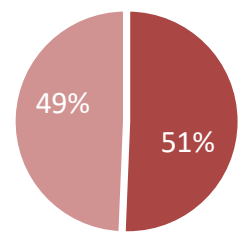

Figura 1. El gráfico representa el porcentajes de AFF que afectan a los Rasgos del fonema y a la Sílaba, respecto del total de AFF producidos por los informantes.
Respecto a la distribución de ocurrencias por tipo de ajuste, se presentan en primer lugar los resultados para los AFF que afectan a los Rasgos (Figura 2) y luego los AFF que afectan a la Sílaba (Figura 3). Considerando el total de la muestra, de los AFF que afectan a los Rasgos (Figura 2), el más frecuente de todos es el Ajuste de Modo con 113 casos, que representa un $48 \%$, seguido de Ajuste de Zona con Lejanía $(\mathrm{ZcL})$ con 34 casos y un $15 \%$, le sigue el Ajuste de Sonoridad con 30 casos y un $13 \%$. Cabe destacar que el Ajuste de Consonante por Vocal, no fue producido por ninguno de los 25 informantes.

\section{AFF A LOS RASGOS: TOTAL DE LA MUESTRA}

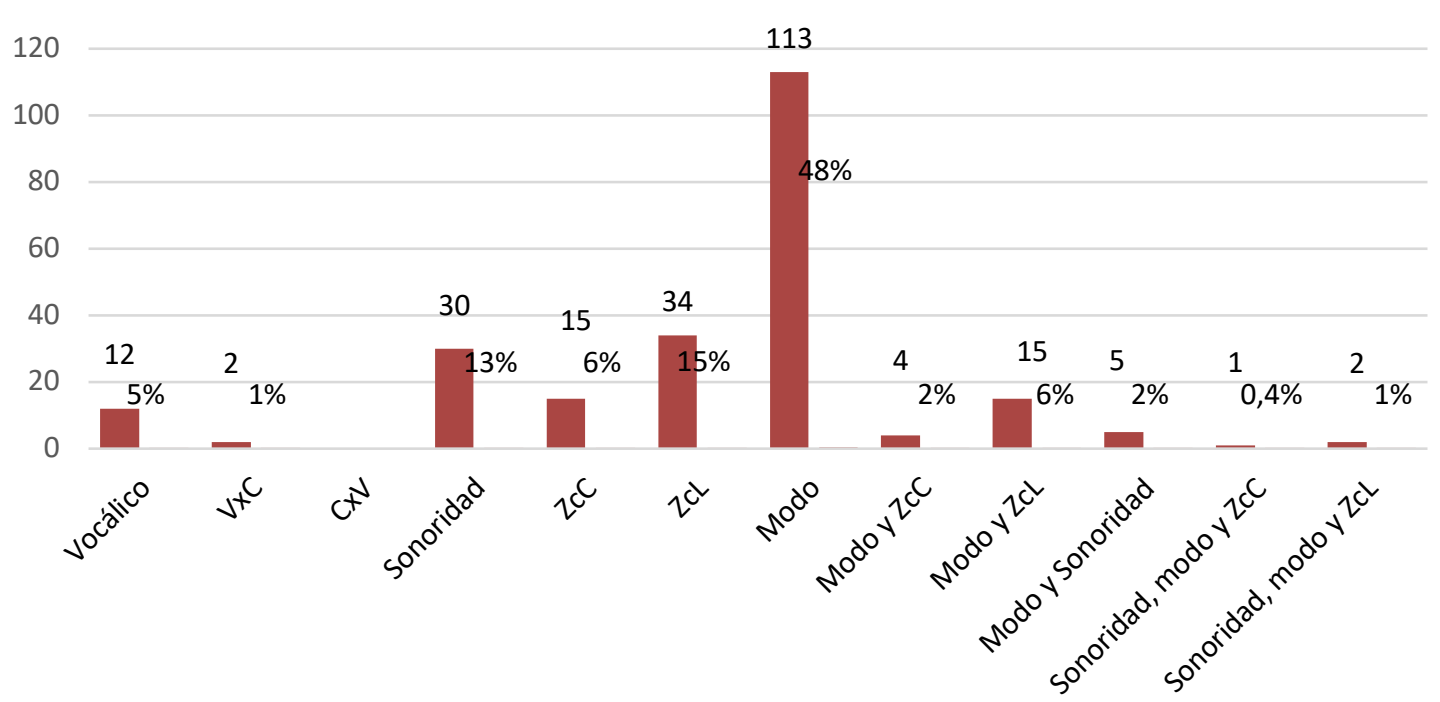

Figura 2. El gráfico representa la frecuencia y porcentaje con que se presentaron los AFF que afectan a los Rasgos, evidenciando la alta ocurrencia del AFF de Modo, seguido por Zona con Lejanía y Sonoridad. Se muestran porcentajes de aparición debajo de cada frecuencia, los valores iguales a $0 \%$ se obviaron de la figura. 
En relación a los AFF que afectan a la Sílaba (Figura 3), considerando el total de los informantes, el AFF de Síncopa (eliminación de sonido en posición interior de palabra) fue el que tuvo mayor frecuencia con 143 casos que representan un 63\%, seguido de
Aféresis (Eliminación de sonido en posición inicial) con 38 ocurrencias y un 17\%, lo sigue el AFF de Epéntesis (Inserción de sonido en posición interior de palabra) con 18 casos y un 8\%. La Diptongación no presentó ocurrencias.

AFF A LA SÍLABA: TOTAL DE LA MUESTRA

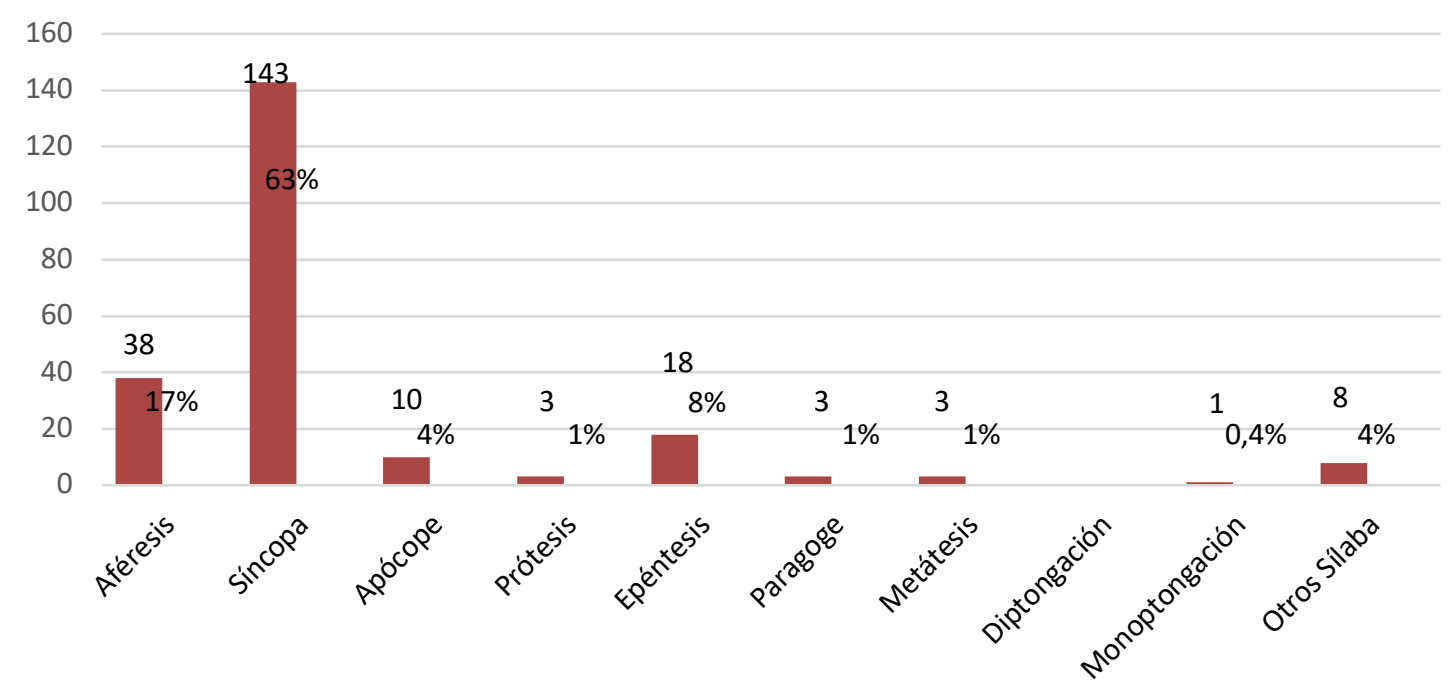

Figura 3. El gráfico representa la frecuencia y porcentaje con que se presentaron los AFF que afectan a la Sílaba, evidenciando la alta ocurrencia del AFF de Síncopa, seguido por Aféresis y Epéntesis. Se muestran porcentajes de aparición debajo de cada frecuencia, los valores iguales a 0 no se presentan en la figura.

Como se puede evidenciar en el gráfico, aparece una nueva categoría llamada "Otros Sílaba", con 7 ocurrencias, en la que los hablantes produjeron [pe.'u.mo] en lugar de /'peu.mo/. En este caso se trata de la disolución de un diptongo, ajuste que no se había registrado en otras poblaciones estudiadas anteriormente.

\section{Análisis estadístico}

Para este análisis se consideraron las variables de Nivel escolar como variable independiente, con sus categorías pre-kínder, kínder, primero, segundo y tercero básicos, y la variable Categoría de ajuste como variable dependiente, con sus niveles de AFF a los Rasgos y AFF a la Sílaba. Como se observa en la Tabla 1, para el nivel pre-kínder el ajuste más frecuente es el Ajuste al Rasgo, mientras que para los niveles kínder y primero básico el ajuste más frecuente es el que afecta a la estructura de la sílaba; esta tendencia se revierte luego para los niveles segundo y tercero básicos, en los que predomina nuevamente el Ajuste al Rasgo. Con la finalidad de explorar la asociación entre las variables Categoría de ajuste y Nivel escolar, se llevó a cabo un análisis de chi-cuadrado, que reveló una asociación estadística significativa entre ambas variables $(\chi 2=$ 16.195, df $=4, p<0.01 ;$ Cramer's $V=0.196)$, con la 
prueba V de Cramer indicando un tamaño de efecto pequeño (Kotrlik, Williams, \& Jabor, 2011).

Para explorar en más detalle esta asociación, se llevó a cabo un análisis de Regresión Logística Multinomial (RLM), con la variable Categoría de ajuste como la variable dependiente con dos niveles (nivel de referencia: sílaba) y Nivel escolar como la variable predictora independiente con cinco niveles (nivel de referencia: primero básico).

Tabla 1

Nivel escolar vs. Categoría de ajustes.

\begin{tabular}{|c|c|c|c|}
\hline & AFF al Rasgo & AFF a la Sílaba & Total \\
\hline Pre-kinder & $\begin{array}{c}48 \\
(58 \%)\end{array}$ & $\begin{array}{c}35 \\
(42 \%)\end{array}$ & $\begin{array}{c}83 \\
(20 \%)\end{array}$ \\
\hline Kinder & $\begin{array}{c}22 \\
(38 \%)\end{array}$ & $\begin{array}{c}36 \\
(62 \%)\end{array}$ & $\begin{array}{c}58 \\
(14 \%)\end{array}$ \\
\hline Primero Básico & $\begin{array}{c}83 \\
(43 \%)\end{array}$ & $\begin{array}{c}110 \\
(57 \%)\end{array}$ & $\begin{array}{c}193 \\
(46 \%)\end{array}$ \\
\hline Segundo Básico & $\begin{array}{c}32 \\
(64 \%)\end{array}$ & $\begin{array}{c}18 \\
(36 \%)\end{array}$ & $\begin{array}{c}50 \\
(12 \%)\end{array}$ \\
\hline Tercero Básico & $\begin{array}{c}25 \\
(64 \%)\end{array}$ & $\begin{array}{c}14 \\
(36 \%)\end{array}$ & $\begin{array}{c}39 \\
(9 \%)\end{array}$ \\
\hline
\end{tabular}

Tabulación cruzada de las variables Categoría de ajuste y Nivel escolar. Se muestran porcentajes de aparición por fila debajo de cada frecuencia, en paréntesis.

Tabla 2

Resumen de los resultados del Análisis Regresión Logística Multinomial.

\begin{tabular}{|c|c|c|c|c|c|c|}
\hline Comparaciones & Estimación (EE) & $p$ & & $\begin{array}{c}\text { IC Inferior } \\
(2.5 \%)\end{array}$ & $\begin{array}{c}\text { Razón de } \\
\text { probabilidades }\end{array}$ & $\begin{array}{c}\text { IC Superior } \\
(97.5 \%)\end{array}$ \\
\hline \multicolumn{7}{|l|}{ Rasgo vs. Sílaba } \\
\hline (Intercept) & $-0.281(0.145)$ & $<0.1$ & . & & & \\
\hline Pre-kinder & $0.597(0.266)$ & $<0.05$ & $*$ & 1.080 & 1.818 & 3.059 \\
\hline Kinder & $-0.211(0.307)$ & $=0.493$ & & 0.444 & 0.810 & 1.479 \\
\hline Segundo Básico & $0.857(0.329)$ & $<0.01$ & $* *$ & 1.237 & 2.356 & 4.486 \\
\hline Tercero Básico & $0.861(0.364)$ & $<0.05$ & $*$ & 1.159 & 2.367 & 4.831 \\
\hline
\end{tabular}

Resultados del análisis RLM con la variable Categoría de Ajuste como variable dependiente y Nivel Escolar como la variable predictora. Se proveen los estimados, su error estándar (EE), su valor de significancia estadística, razones de probabilidades e intervalos de confianza para las razones de probabilidades. Niveles de significancia: $* * *<0.001, * *<0.01, *<0.05, .<0.1$. Log-Likelihood (Función de verosimilitud) $=-285.02$. McFadden R2 (Pseudo R-cuadrado): 0.028. Likelihood ratio test (Prueba de razón de verosimilitud): $\chi 2=16.344, p<0.01$. 
Los resultados mostraron que la variable Nivel escolar es capaz de predecir la probabilidad de aparición del nivel "rasgo" por sobre el de "sílaba", con significancia estadística, para los niveles prekínder, segundo básico y tercero básico (véase Tabla 2), pero no para kínder. En particular, es casi dos veces más probable que en el nivel pre-kínder aparezca un ajuste de "rasgo" en lugar de uno de "sílaba", con una razón de probabilidad de 1,818. Además, es varias veces más probable que "rasgo" surja en lugar de "sílaba" en los niveles segundo y tercero básicos, con una razón de probabilidad de 2,356 y 2,367 , respectivamente.

\section{Discusión}

Considerando los resultados para el total de la muestra, es posible afirmar que los niños con TEA presentan dos marcadas tendencias de estrategias para modificar las palabras considerando los tipos de ajustes. La primera de ellas es la tendencia a ajustar el Modo de articulación de los fonemas como forma de ajuste sobre los rasgos, y la segunda la tendencia a producir Síncopa o eliminar sonidos que se encuentran dentro de la palabra como método de ajuste a la estructura de la sílaba. Los hallazgos descritos pueden ser interpretados como alteraciones del nivel fonético-fonológico en los niños con TEA que cursaban los niveles de primero, segundo y tercero básico. Esto debido a que se trata de un rango etario en el cual el desarrollo fonológico ya debiera haberse completado en niños chilenos (Pavez et al., 2009; Vivar \& León, 2009). Los AFF producidos por los niños de kínder y prekínder, por ahora, solo pueden ser interpretados como manifestaciones del desarrollo fonológico, puesto que la metodología empleada en el presente estudio no permite efectuar comparaciones con otros precedentes investigativos.

El hallazgo de alteraciones fonológicas en los sujetos de la presente investigación, concuerda con estudios recientes realizados para la lengua inglesa que reportan la ocurrencia de distorsiones fonológicas de diverso tipo y severidad en personas con TEA. Los resultados de estos estudios contrastan con la posición más difundida que afirmaba que el nivel fonético-fonológico no presenta grandes alteraciones en personas con esta condición (Happé, 1998; McCann et al., 2007). Además, los hallazgos del presente estudio están en línea con lo observado más tempranamente por Shriberg et al. (2001), quienes encontraron una alta prevalencia de distorsión de sonidos del habla en adolescentes y adultos con Autismo de Alto Funcionamiento (AAF) y Síndrome de Asperger (SA), quienes modificaban la zona y el modo de articulación. Este fenómeno es bastante similar al observado en la presente investigación, en la que se evidenció el Ajuste de Modo como el más frecuente, seguido del Ajuste de Zona con lejanía. Shriberg et al. (2001) señalan que estas dificultades en la población con TEA pueden ser un reflejo de la falla del hablante al utilizar recursos para producir un habla precisa que coincida con el modelo esperado. Una explicación probable es la existencia de alteraciones motoras finas y gruesas en las personas con TEA, como las reportadas por Lloyd, MacDonald, \& Lord (2013), las cuales se acentuarían con la edad. Otra posible explicación a las dificultades fonético-fonológicas de niños con TEA encontradas en esta investigación, surge del estudio 
de You, Serniclaes, Rider, \& Chabane (2017). Este estudio señala que las personas con TEA presentan dificultades en la percepción del habla. Estas dificultades se manifestarían como un descenso en la precisión de la percepción de las características fonológicas de su lengua nativa. Lo anterior se evidenció por un descenso en la percepción categorial fonológica de vocales y consonantes por lugar de articulación en comparación con niños con desarrollo típico del lenguaje. Esto también podría tener cierta relación con las características propias del TEA, como la existencia de intereses fijos y comportamientos repetitivos, que se asocian a déficits neurocognitivos del comportamiento flexible (D'Cruz et al., 2013) y en las dificultades de pensamiento abstracto (Minshew, Meyer, \& Goldstein, 2002) que podrían inducir dificultades fonológicas derivadas del manejo de elementos abstractos como los fonemas.

Ahora bien, si se consideran las categorías de ajuste para el total de la muestra, a saber, AFF a los rasgos y AFF a la sílaba, no se observa alguna preferencia por alguno de ellos, puesto que los ajustes se distribuyeron de manera equitativa entre ambas categorías, con $51 \%$ para los AFF a los rasgos y $49 \%$ para los AFF a la sílaba. Sin embargo, si se consideran los datos entregados por la tabla de contingencias (Tabla 1) que compara Nivel educativo y Categoría de ajuste, junto con los análisis estadísticos realizados, tanto la prueba de chicuadrado con el análisis de Regresión Logística Multinomial (Tabla 2) se observa que existe una tendencia a modificar los Rasgos en los niveles prekínder, segundo y tercero básico. Esta dificultad en el manejo de los rasgos, evidenciada por la tendencia a modificarlos, podría ser explicada por la dificultad motora que presentan los niños con TEA, señalada por (Lloyd et al., 2013). Sin embargo, en los niveles kínder y primero básico, la preferencia se invierte surgiendo como primera opción el ajuste a la sílaba por sobre el ajuste a los rasgos. Una explicación posible para este hecho puede ser la influencia del proceso lecto-escritor de la enseñanza escolar formal, y su impacto en el nivel fonológico de los sujetos. Las Bases Curriculares que entrega el Ministerio de Educación hacen mención a que en kínder debe darse la iniciación a la lectura y a la escritura (Ministerio de Educación, 2005) y en primero básico la organización curricular de “Lenguaje y Comunicación" presenta tres ejes, uno de los cuales es Lectura y otro Escritura (Ministerio de Educación, 2013). Esto sin duda representa una alta exigencia de desarrollo de habilidades fonológicas, ya que el niño debe segmentar los distintos elementos y asignar a cada uno de ellos un sonido para lograr el proceso de lectoescritura de una palabra (López, 2016), lo que para una población como los niños con TEA representa una importante dificultad al presentar bajas habilidades de percepción del habla (You et al., 2017).

Otro hecho interesante observado, es que el tipo de AFF producido exhibe tendencias que se asemejan a las de estudios anteriores para niños chilenos con desarrollo típico (León, 2012) y TEL mixto (Torres et al., 2016). Es decir, un leve predominio de los AFF que afectan a los rasgos por sobre los de estructura silábica y mayor utilización del AFF de Modo y Síncopa, en los AFF a los rasgos y a la estructura silábica respectivamente. Sin embargo, se encuentran diferencias con 
investigaciones previas en el uso reportado del AFF de $Z c L$ en esta población, el que aparece como segunda opción de ajuste a los rasgos, fenómeno antes no observado. Adicionalmente, podemos observar un leve aumento de la utilización del AFF de Aféresis como segunda opción en los AFF que afectan la estructura silábica.

\section{Conclusiones}

Los hallazgos descritos permiten señalar que los niños con TEA estudiados realizaban principalmente dos tipos de AFF: A los rasgos del fonema (Ajuste de Modo) y a la sílaba (Síncopa). La presencia de estos AFF puede ser interpretados como alteraciones del nivel fonético fonológico en los participantes que cursan los niveles de primero, segundo y tercero básico debido a que se espera que este nivel se encuentre desarrollado en esta etapa (Pavez et al., 2009; Vivar \& León, 2009).

Al observar la totalidad de AFF efectuados, se puede evidenciar que las estrategias de ajuste utilizadas por los niños con TEA coinciden en sus primeras opciones con las reportadas por otros estudios realizados en niños chilenos con desarrollo típico (León, 2012) y con diagnóstico de TEL mixto (Torres et al., 2016), tanto en los ajustes que predominaron para las modificaciones a los rasgos como a la sílaba.

Cabe destacar, a partir de lo presentado, que las dificultades fonológicas de los niños estudiados no son desestimables e implican un obstáculo importante en su comunicación oral, lo que debe ser estudiado más profundamente y considerado en la planificación de los apoyos requeridos por esta población. No se puede desconocer que las dificultades en este ámbito repercuten en el rendimiento educativo y social de estos niños, y especialmente ante los desafíos que presentan quienes participan de un modelo de educación inclusiva.

Finalmente, se requiere mayor profundización en el estudio del desempeño fonético fonológico de los niños con TEA. En subsecuentes estudios, se debe intentar aumentar el tamaño muestral y equilibrar el número de participantes por nivel de manera de facilitar los análisis que permitan comparar y establecer la significancia estadística de los hallazgos.

\section{Referencias}

American Psychiatric Association. (2014). Manual Diagnstico y Estadstico de los Trastornos Mentales (DSMV) (5.a ed.). Arlington, Estados Unidos: American Psychiatric Association.

American Psychiatric Association, \& López-lbor Aliño, J. J. (2002). Manual diagnóstico y estadístico de los trastornos mentales (DSM-IV-TR). Barcelona, España: Masson.

Asperger, H. (1944). Die Autistischen Psychopathen im Kindesalter. Archiv Für Psychiatrie Und Nervenkrankheiten, 117(1), 76-136.

Boersma, P., \& Weenink, D. (2018). Praat: doing phonetics by computer (Versión 6.0.39). Recuperado de http://www.praat.org/

Cleland, J., Gibbon, F. E., Peppé, S. J. E., O'Hare, A., \& Rutherford, M. (2010). Phonetic and phonological errors in children with high functioning autism and Asperger syndrome. International Journal of Speech-Language Pathology, 12(1), 69-76.

D'Cruz, A.-M., Ragozzino, M. E., Mosconi, M. W., Shrestha, S., Cook, E. H., \& Sweeney, J. A. (2013). Reduced behavioral flexibility in autism spectrum disorders. Neuropsychology, 27(2), 152-160. https://doi.org/10.1037/a0031721 
Dioses, A., Matalinares, M., Velásquez, C., Cuzcano, A., Chávez, J., Campos, M., ... Solórzano, A. (2014). Lenguaje oral en niños con trastornos del espectro autista. Theorema (Lima, Segunda Época, En línea), 1(1), 111-122.

Fortea, M., Escandell, M., \& Castro, J. J. (2014). Nuevas formas de abordaje del proceso diagnóstico del TEA después del DSM-5. International Journal Of Developmental And Educational Psychology, 1(1), 77-86.

Fuica, M. A., \& Soto-Barba, J. (2014). Ajustes fonéticofonológicos en niños(as) de 4 a 5 años que aprenden inglés como segunda lengua. Literatura y lingüística, (30), 258283. https://doi.org/10.4067/S0716-58112014000200014

Gerber, S. (2003). A Developmental Perspective On Language Assessment And Intervention For Children On The Autistic Spectrum. Topics in Language Disorders, 23(2), 74-79. https://doi.org/10.1097/00011363-20030400000003

Hamdan, N. (2017). Desarrollo fonético-fonológico y léxicosemántico de preescolares y su relación con el desempeño linguístico de sus educadoras de párvulos (Tesis de Magister). Universidad de Concepción. Facultad de Humanidades y Arte, Concepción, Chile. Recuperado de http://repositorio.udec.cl/handle/11594/2633

Happé, F. (1998). Introducción al autismo. Madrid: Alianza Editorial.

Irarrázaval, M., Brokering, W., Murillo, G., B, M., \& Adolfo, G. (2005). Autismo: una mirada desde la psiquiatría de adultos. Revista chilena de neuro-psiquiatría, 43(1), 51-60. https://doi.org/10.4067/S0717-92272005000100007

Kanner, L. (1943). Autistic disturbances of affective contact. Nervous Child, 2, 217-250.

Kotrlik, J., Williams, H., \& Jabor, M. K. (2011). Reporting and Interpreting Effect Size in Quantitative Agricultural Education Research. Journal of Agricultural Education, 52(1), 132-142. https://doi.org/10.5032/jae.2011.01132

León, H. (2012). Ajustes fonético-fonológicos en niños(as) de habla normal entre 3 a 4 años y 4 a 5 años (Tesis Doctoral). Universidad de Concepción, Concepción, Chile. Recuperado de http://repositorio.udec.cl/handle/11594/760
Lloyd, M., MacDonald, M., \& Lord, C. (2013). Motor Skills of Toddlers with Autism Spectrum Disorders. Autism : the international journal of research and practice, 17(2), 133146. https://doi.org/10.1177/1362361311402230

López, L. (2016). Conciencia fonológica, memoria fonológica y habilidades lectoras en escolares dados de alta de TEL y escolares con desarrollo típico del lenguaje de primer año de educación básica (Tesis de Magíster). Universidad Católica de la Santísima Concepción, Concepción, Chile.

McCann, J., Peppé, S., Gibbon, F. E., O'Hare, A., \& Rutherford, M. (2007). Prosody and its relationship to language in school-aged children with high-functioning autism. International Journal of Language \& Communication Disorders, 42(6), 682-702. https://doi.org/10.1080/13682820601170102

McCleery, J. P., Tully, L., Slevc, L. R., \& Schreibman, L. (2006). Consonant production patterns of young severely language-delayed children with autism. Journal of Communication Disorders, 39(3), 217-231. https://doi.org/10.1016/j.jcomdis.2005.12.002

Ministerio de Educación. (2005). Bases Curriculares de la Educación Parvularia (1.a ed.). Santiago, Chile.

Ministerio de Educación. (2013). Lenguaje y Comunicación, Programa de Estudio para Primer Año Básico (1.a ed.). Santiago, Chile.

Minshew, N. J., Meyer, J., \& Goldstein, G. (2002). Abstract reasoning in autism: a dissociation between concept formation and concept identification. Neuropsychology, 16(3), 327-334.

Organización Mundial de la Salud (Ed.). (1992). Clasificación Internacional de las Enfermedades. Trastornos mentales y del comportamiento. Descripción clínica y pautas para el diagnóstico CIE-10 (Décima revisión. [10a rev.]). Madrid: Méditor.

Pavez, M. M., Maggiolo, M., Peñaloza, C., \& Coloma, C. J. (2009). Desarrollo fonológico en niños de 3 a 6 años: incidencia de la edad, el género y el nivel socioeconómico. RLA: Revista de lingüística teórica y aplicada, 47(2), 89-109.

R Core Team. (2018). R: A language and environment for statistical computing (Versión 3.4.4). 
Rapin, I., Dunn, M. A., Allen, D. A., Stevens, M. C., \& Fein, D. (2009). Subtypes of language disorders in school-age children with autism. Developmental Neuropsychology, 34(1),

66-84.

https://doi.org/10.1080/87565640802564648

Rodríguez, J. M. (1988). Aspectos formales del lenguaje en el autismo infantil: I. fonología. Revista de Logopedia, Foniatría y Audiología, 8(1), 23-29. https://doi.org/10.1016/S0214-4603(88)75422-4

Seung, H. K. (2007). Linguistic characteristics of individuals with high functioning autism and Asperger syndrome. Clinical Linguistics \& Phonetics, 21(4), 247-259. https://doi.org/10.1080/02699200701195081

Shriberg, L. D., Paul, R., McSweeny, J. L., Klin, A., Cohen, D. J., \& Volkmar, F. R. (2001). Speech and Prosody Characteristics of Adolescents and Adults With HighFunctioning Autism and Asperger Syndrome. Journal of Speech, Language, and Hearing Research, 44(5), $1097-$ 1115. https://doi.org/10.1044/1092-4388(2001/087)

Soto-Barba, J., León, H., \& Torres, V. (2011). Una propuesta para la clasificación de los ajustes fonético-fonológicos del habla infantil (CLAFF). Onomázein, 23(1), 69-79.

Torres, V., Soto-Barba, J., Soto-Barba, J., \& Universidad de Concepción. (2016). Ajustes fonético-fonológicos en niños con trastornos específicos del lenguaje mixto (TEL Mixto). Onomázein, 33(1), 69-87. https://doi.org/10.7764/onomazein.33.7

Vivar, P., \& León, H. (2009). Desarrollo fonológico-fonético en un grupo de niños entre 3 y 5, 11 años. Revista CEFAC, 11(2), 190-198. https://doi.org/10.1590/S151618462009000200003

Westerveld, M. F., \& Roberts, J. M. A. (2017). The Oral Narrative Comprehension and Production Abilities of Verbal Preschoolers on the Autism Spectrum. Language, Speech, and Hearing Services in Schools, 48(4), 260-272. https://doi.org/10.1044/2017_LSHSS-17-0003
Whitehouse, A., Barry, J., \& Bishop, D. (2008). Further defining the language impairment of autism: is there a specific language impairment subtype? Journal of Communication Disorders, 41(4), 319-336. https://doi.org/10.1016/j.jcomdis.2008.01.002

Wing, L., \& Gould, J. (1979). Severe impairments of social interaction and associated abnormalities in children: epidemiology and classification. Journal of Autism and Developmental Disorders, 9(1), 11-29.

Wing, Lorna. (1997). The autistic spectrum. The Lancet, 350(9093), 1761-1766. https://doi.org/10.1016/S01406736(97)09218-0

Wing, Lorna. (1998). El autismo en niños y adultos: Una guía para la familia. Madrid: Ediciones Paidós Ibérica.

You, R. S., Serniclaes, W., Rider, D., \& Chabane, N. (2017). On the nature of the speech perception deficits in children with autism spectrum disorders. Research in Developmental Disabilities, 61, 158-171. https://doi.org/10.1016/j.ridd.2016.12.009 


\section{Anexo 1}

\section{Consentimiento Informado para Padre/Madre de los Participantes en el estudio}

“Estudio del desempeño fonético-fonológico de niños con Trastorno del Espectro Autista (TEA) de los niveles prekínder, kínder, primero, segundo y tercero básico en Programas de Integración Escolar (PIE) en establecimientos del DAEM de Talcahuano"

\section{Protocolo de información \\ para el padre o madre del o la participante}

Estimado(a) Señor(a),

El propósito de este consentimiento es entregar información clara acerca de la naturaleza de este trabajo de investigación para optar al grado de Magíster en Lingüística Aplicada y del rol que tendrán en ella los participantes. Así, Uds. padres/madres o tutores podrán decidir, a conciencia, si desean que sus hijos(as) o pupilo participen o no. A continuación se le proporcionará toda la información para leer detenidamente antes de que se decida a autorizar o no la participación en dicho estudio.

No dude en preguntar al investigador responsable si tiene alguna duda o necesita alguna aclaración sea ésta antes, durante o después de leer este documento.

\section{INTRODUCCIÓN}

La investigación corresponde al desarrollo de la tesis "Estudio del desempeño fonético-fonológico de niños con Trastorno del Espectro Autista (TEA) de los niveles pre-kínder, kínder, primero, segundo y tercero básico en Programas de Integración Escolar (PIE) en establecimientos del DAEM de Talcahuano", para optar al grado de Magíster en Lingüística Aplicada del Fonoaudiólogo Juan Carlos Ernesto Torres Torres, a cargo del Profesor Dr. Hernán León.

\section{OBJETIVO}

El objetivo de esta investigación se orienta a describir el lenguaje oral de estudiantes que sean parte de Programas de Integración Escolar bajo el diagnóstico de Trastorno del Espectro Autista o Síndrome de Asperger.

Para llevar a cabo esta investigación, se requiere de la participación de alumnos(as) desde pre-kínder a tercero básico de establecimientos educacionales dependientes del DAEM de Talcahuano con el diagnóstico señalado.

\section{PROCEDIMIENTO}

La colaboración de su hijo/a o pupilo consistirá en que él o ella participe en una entrevista en donde se le pedirá lo siguiente:

a. Participar en un examen de sus órganos del habla (cara, boca y cuello), y

b. Participar en una prueba de repetición de palabras.

Toda la conversación será grabada en audio. Además, estas actividades se realizarán en dependencias del establecimiento educacional al que asiste su hijo, según autorización de su Director(a), y tendrán una duración aproximada de 15 minutos.

\section{BENEFICIOS}

Se espera que la participación de su hijo(a) o pupilo en este estudio contribuya a comprender más y mejor cómo los niños y niñas con diagnóstico de Trastorno del Espectro Autista o Síndrome de Asperger que son parte de Programas de Integración Escolar, usan el lenguaje oral. 


\section{RIESGOS}

En el estudio que se llevará a cabo, no se identifica ningún riesgo para la salud de su hijo(a) o pupilo, ni tendrá consecuencia negativa producto de su participación.

\section{CONFIDENCIALIDAD}

La información que se recoja será confidencial y no se usará para ningún otro propósito que no sean los propios de esta investigación. Sus respuestas serán tratadas de forma anónima. Los resultados obtenidos serán entregados al fonoaudiólogo del establecimiento al término del proyecto, y si usted lo requiere podrá solicitarlos. El Investigador Responsable custodiará los datos de este estudio, identificando con claves los nombres de cada participante y resguardando la información en su computador.

\section{COSTOS}

Su participación no tendrá costo alguno para usted.

\section{COMPENSACIÓN}

Usted o su hijo(a)/ pupilo no recibirá compensación económica por la participación en este estudio

\section{DERECHO A RETIRARSE DEL ESTUDIO}

La participación de su hijo(a) en este estudio es libre y voluntaria. Ud. tiene derecho a retirar a su hijo(a)/ pupilo del estudio en cualquier momento y su decisión no afectará, bajo ningún concepto, sus actividades escolares o su relación con los investigadores o profesores.

\section{CONTACTO}

Si tiene alguna duda sobre este proyecto, puede hacer consultas al Investigador Responsable, Fonoaudiólogo Juan Carlos Torres (jtorrest@udec.cl).

Si usted tiene alguna pregunta acerca de los derechos como participante en esta investigación o siente vulnerados sus derechos, usted puede llamar al Presidente u otro miembro del Comité de Ética, Bioética y Bioseguridad de la Vicerrectoría de Investigación y Desarrollo de la Universidad de Concepción, Dr. José Becerra Allende, al fono: (41) 2204302. 


\section{DECLARACIÓN DE CONSENTIMIENTO INFORMADO}

Yo, D./Dña. autorizo a mi hijo(a)/ pupilo a participar de esta investigación titulada "Estudio del desempeño fonético-fonológico de niños con Trastorno del Espectro Autista (TEA) de los niveles pre-kínder, kínder, primero, segundo y tercero básico en Programas de Integración Escolar (PIE) en establecimientos del DAEM de Talcahuano".

Declaro que he leído y entendido la información que se me ha entregado.

He recibido suficiente información sobre el estudio.

He podido hacer preguntas sobre las características del estudio.

Comprendo que la participación de mi hijo(a)/ pupilo es libre y voluntaria.

Comprendo que éste puede retirarse del estudio en cualquier momento.

Presto libre y voluntariamente mi conformidad para su participación. Por ello, autorizo y doy mi consentimiento de manera libre e informada para que mi hijo(a)/ pupilo participe en este estudio.

Entiendo que una copia de este documento de consentimiento me será entregada, y que puedo pedir información sobre los resultados del estudio cuando éste haya concluido.

Fecha

Nombre y firma de/ de la Apoderado(a)

Nombre y firma Investigador Responsable

Nombre y firma Director del establecimiento o su delegado/Ministro de fe

Agradecemos la participación de su hijo(a). 
Anexo 2

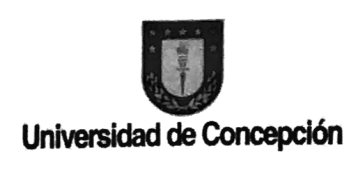

Concepción, Enero de 2018.

\author{
CERTIFICADO
}

El Comité de Ética, Bioética y Bioseguridad de la Vicerrectoría de Investigación y Desarrollo de la Universidad de Concepción ha revisado el protocolo del Proyecto de Tesis para optar al grado de Magíster en Lingüística, titulado "ESTUDIO DEL DESEMPEÑO FONÉTICO-FONOLÓGICO DE NIÑOS CON TRASTORNO DEL ESPECTRO AUTISTA DE LOS NIVELES PRE-KÍNDER A TERCERO BÁSICO EN PROGRAMAS DE INTEGRACIÓN ESCOLAR DEL DAEM DE TALCAHUANO" postulado por el fonoaudiólogo SR. JUAN CARLOS ERNESTO TORRES TORRES, en calidad de candidato al grado de Magíster en Lingüística, junto a su Profesor Guía DR. HERNÁN LEÓN VALDÉS, adscrito al Departamento de Fonoaudiología de la Facultad de Medicina de la Universidad de Concepción, y ha comprobado que cumple con las normas y procedimientos éticos y bioéticos establecidos nacional e internacionalmente para estudios que involucran personas.

La presente propuesta de Proyecto de Tesis para la obtención del grado de Magister en Lingüistica por la Universidad de Concepción, tiene como objetivo analizar el desempeño fonético-fonológico de niños con Trastorno del Espectro Autista (TEA) de los niveles pre-kínder, kinder, primero, segundo y tercero básico que se encuentren en Programas de Integración Escolar (PIE) en establecimientos dependientes del Departamento de Administración de Educación Municipal (DAEM) de Talcahuano, mediante el uso de la Clasificación de Ajustes Fonético-Fonológicos del habla infantil (CLAFF). Para ello, pretende desarrollar 05 (cinco) objetivos específicos. Al inicio prevé evaluar la producción de ajustes fonético-fonológicos (AFF) de niños con TEA de prekínder, kínder, primero, segundo y tercero básico en PIE. Después pretende describir esta producción de AFF y categorizar los tipos de AFF que realizan los niños con TEA de pre-kínder, kínder, primero, segundo y tercero básico en PIE. Con estos datos, tiene previsto contrastar los tipos de AFF que realizan los niños con TEA de pre-kínder, kínder, primero, segundo y tercero básico en PIE con los datos de otras patologías del lenguaje para, finalmente, determinar si el desempeño fonético-fonológico de estos niños con diagnóstico TEA es propio de la patología o es concomitante a la misma. Todo lo anterior está rigurosamente detallado en "Metodología", incluyendo los métodos de investigación que contemplan la selección de muestra, la aplicación de los instrumentos, los análisis parciales y finales y la elaboración de las conclusiones.

En este estudio, la propuesta metodológica contempla seleccionar a 36 (treinta seis) individuos (informantes) que cumplan con los criterios de inclusión descritos ef Proyecto de Tesis. 


\section{Ui \\ Universidad de Concepción}

La aplicación de los instrumentos de evaluación para cada actividad del estudio se realizará en el establecimiento educacional a que pertenezca el/ la niño(a), por el Investigador Responsable, Sr. Juan Carlos Ernesto Torres Torres, acompañado de la profesora encargada del curso.

La participación de cada sujeto seleccionado estará basada en el proceso de Asentimiento Informado (verbal) y de Consentimiento Informado para los padres/ madres - tutor, toda vez que este último sea regularmente firmado. Casa proceso de Consentimiento Informado será documentado, conforme modelos presentados a este Comité institucional.

La custodia de la información y de los resultados del estudio que se propone, será responsabilidad del estudiante tesista, Sr. Juan Carlos Ernesto Torres Torres.

La ejecución de esta investigación científica en el Proyecto de Tesis en pauta asegura que no vulnera los derechos y la dignidad de los sujetos participantes en la investigación, garantizando la voluntariedad y la privacidad de los mismos, presentando para ello los métodos de protección que aseguran la confidencialidad de los datos de investigación y de custodia estricta de la información obtenida (modelo de documento escrito de "Consentimiento Informado"), observando todas las características formales y necesarias para su validez.

Este Comité considera que el Proyecto presentado observa los derechos asegurados en la Declaración Universal de los Derechos Humanos, los derechos y principios de la Declaración Universal sobre Bioética y Derechos Humanos, las Normas Éticas de la Organización Panamericana de la Salud para Investigaciones con Sujetos Humanos, la Constitución de la República de Chile, la Ley $\mathrm{N}^{\circ} 20.120$ "Sobre la Investigación Científica en el Ser Humano, su Genoma y Prohíbe la Clonación Humana", la Ley $\mathrm{N}^{\circ}$. 20.584, que regula los derechos y deberes que tienen las personas en relación con acciones vinculadas a su atención en salud. Así también, sigue las Sugerencias para Escribir un Consentimiento Informado en Estudios con Personas, del Comité Asesor de Bioética FONDECYT/CONICYT.

En atención a lo anterior y dado que el Proyecto de Tesis presentado no muestra elementos que puedan transgredir las normas y principios éticos y bioéticos de la investigación en seres humanos, así como también los principios rectores de nuestra Institución Universitaria, los delineados en la Declaración de Singapur sobre la Integridad en la Investigación (2010) y las normas adoptadas por la Comisión Naciopa $1 \mathrm{de}$ Investigación Científica y Tecnológica - CONICYT, este Comité resuelve aptobarlo confiriendo el presente Certificado.

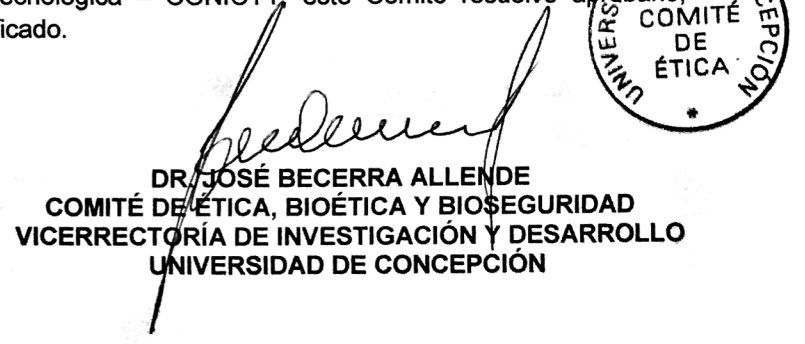

